\section{Seltene Karzinome von Ovar und Peritoneum}

\section{Seröse Low-grade-Tumoren von Ovar oder Peritoneum sind selten, betreffen vornehmlich jüngere Frauen und zeichnen sich durch relative Chemothera-} pieresistenz aus. Eine retrospektive US-Studie gibt Einblick in den Zusammenhang zwischen Erkrankungsalter, primärer Tumorlokalisation und Überleben.

V or gut 10 Jahren entwickelte die Arbeitsgruppe um David M. Gershenson das Konzept einer binären Graduierung für seröse Low-grade-Karzinome von Ovar (LGSOC) oder Peritoneum (LGSPC), das mittlerweile breite Akzeptanz findet. Im Jahr 2007 legten Gershenson und Kollegen ein Register an, in das systematisch demografische und klinische Informationen aller Frauen mit solchen Tumoren eingingen. Nun präsentieren sie ihre Erfahrungen mit einer größeren Patientinnenkohorte.

350 Frauen erfüllten die Eingangskriterien: LGSOC oder LGSPC der Stadien I-IV, Erstdiagnose vor Januar 2012 und ausreichende klinische Informationen.
Die Mehrzahl der Patientinnen durchlief postoperativ eine Chemotherapie. Hauptendpunkte waren progressionsfreies Überleben (PFS) und Gesamtüberleben (OS). In einer Subgruppenanalyse wurden Patientinnen in den Stadien IIIV erfasst, die nach primärer Tumorexzision eine platinbasierte Chemotherapie erhalten hatten.

Nach median rund 6 Jahren lag das mediane PFS bei 28,1 Monaten und das OS bei 101,7. Jüngeres Erkrankungsalter scheint mit einer schlechteren Prognose verbunden zu sein: Bei Patientinnen mit der Erstdiagnose nach dem 35. Lebensjahr war die Wahrscheinlichkeit, an dieser Erkankung zu versterben, im Ver- gleich zu jüngeren um $43 \%$ geringer. $\mathrm{Pa}$ tientinnen, die nach der Primärtherapie nicht krankheitsfrei sind, müssen mit einer um $78 \%$ höheren Mortalität rechnen als Frauen mit klinisch nicht manifester Erkrankung.

Die Subgruppenanalysen belegten zudem ein reduziertes Mortalitätsrisiko (41\%) für Frauen mit einem LGSPC gegenüber Patientinnen mit LGSOC.

Fazit: Jüngeres Alter und persistierende Krankheit nach Ersttherapie implizieren für Frauen mit serösem Low-GradeKarzinom von Ovar oder Peritoneum eine sehr schlechte Prognose. Findet das maligne Geschehen ausschließlich im Peritoneum statt, scheint die Prognose besser.

Wolfgang Zimmermann

Gershenson DM et al. Impact of age and primary disease site on outcome in women with low-grade serous carcinoma of the ovary or peritoneum: results of a large single-institution registry of a rare tumor. J Clin Oncol. 2015;33(24):2675-82

\title{
Intraperitoneale Therapie beim Ovarialkarzinom: zu selten eingesetzt
}

\section{Patientinnen mit Ovarialkarzinom haben einen Überlebensvorteil, wenn sie die Chemotherapie intra- peritoneal und intravenös erhalten. Unklar war bisher, wie gut diese Erkenntnis außerhalb von Studien umgesetzt wird.}

n mehreren klinischen Studien konnte bereits gezeigt werden, dass die intravenöse (i.v.) in Kombination mit der intraperitonealen (i.p.) Chemotherapie die Überlebensrate von Patientinnen mit einem Ovarialkarzinom im Stadium III nach optimaler Resektion im Vergleich zur alleinigen i.v.-Therapie verbessert. In der GOG-172-Studie etwa wurde mit Paclitaxel und Oxaliplatin eine Verbesserung der Überlebenszeit um 16 Monate erzielt. Daraufhin ermutigte das US National Cancer Institute im Jahr 2006 zur Anwendung der Kombinationsstrategie.

In einer aktuellen prospektiven Beobachtungsstudie wurde unter anderem geschaut, wie sich die Anwendungshäu- figkeit dieser Kombinationstherapie mit den Jahren veränderte und welchen Einfluss sie auf das Gesamtüberleben hatte. Ausgewertet wurden Daten von insgesamt 823 Patientinnen, die zwischen 2003 und 2012 behandelt worden waren. Nach Ausschluss jener Patientinnen, die an klinischen Studien teilgenommen hatten, wurden schließlich die Daten von 498 Patientinnen für die Berechnung der Gesamtüberlebensrate genutzt.

Tatsächlich nahm zwar der Anteil der i.v./i.p.-Therapien im Vergleich zu anderen Strategien zwischen 2003 und 2006 an 6 ausgewählten Krebszentren von 0 auf $33 \%$ zu und zwischen 2007 und 2008 noch einmal auf $50 \%$. Danach stieg die Rate jedoch nicht weiter. Dabei war bis 2012 der Anteil dieser Therapiestrategie im Vergleich zu anderen Behandlungsoptionen in den Zentren sehr unterschiedlich und reichte von 4 bis $67 \%$. Bei $43 \%$ der i.v./i.p.-Therapien variierten die Behandler zudem das ursprünglich in der GOG-172-Studie erprobte therapeutische Vorgehen.
Der Effekt der i.v./i.p.-Therapie auf den Parameter Gesamtüberleben war ähnlich wie in bisherigen Studien. So wurde das Sterberisiko durch das kombinierte Vorgehen im Vergleich zur alleinigen i.v.-Applikation um $32 \%$ gesenkt (relatives Risiko 0,68, 95\%-Konfidenzintervall 0,47-0,99). In der GOG172-Studie lag dieser Wert bei 0,75.

Fazit: In der Beobachtungsstudie konnte erstmals auch außerhalb klinischer Untersuchungen gezeigt werden, dass die kombinierte i.v./i.p.-Therapie Patientinnen mit Ovarialkarzinom einen Überlebensvorteil bietet, entsprechend etwa dem, der in klinischen Studien beobachtet wurde. Jedoch wird diese Strategie - zumindest in den USA - mit einem Anteil von nur $41 \%$ bei denjenigen, die dafür infrage kämen, noch viel zu wenig genutzt.

Peter Leiner

Wright AA et al. Use and Effectiveness of Intraperitoneal Chemotherapy for Treatment of Ovarian Cancer. J Clin Oncol. 2015;33(26):2841-7. 